BUHEP-97-25

RU-97-70

YCTP-P16-97

\title{
The Field Theory of Non-Supersymmetric Brane Configurations
}

\author{
Nick Evans* \\ Department of Physics, Boston University, Boston, MA 02215 \\ and \\ Myckola Schwetz ${ }^{\dagger}$ \\ Department of Physics, Yale University, New Haven, CT 06520-8120 \\ and \\ Department of Physics and Astronomy, Rutgers University, Piscataway, NJ 08855-0849
}

\begin{abstract}
We identify the 4D field theories living on the world volume of D4 branes in nonsupersymmetric type IIA string theory constructions. They are softly broken $\mathrm{N}=2$ SQCD with the breakings introduced through vevs of the auxilliary fields in the spurion coupling field. Exact solutions of these theories for perturbing soft breakings exist in the literature. We calculate the ratios of string tensions in softly broken $\mathrm{N}=2 \mathrm{SU}(\mathrm{N})$ gauge theory testing the recently proposed M-theory prediction. The semi-classical result of M-theory is renormalized in the non-supersymmetric models.
\end{abstract}

*nevans@physics.bu.edu

†myckola@baobab.rutgers.edu 


\section{Introduction}

The latest twist in the tale of the deciphering of supersymmetric gauge theories has been provided by string theory. It has been realized that 4D gauge theories may be constructed as the effective theory on the world volume of D-branes in string theory [1] [2]-12] (an alternative realization of the field theory dualities in string theory has been investigated in [13]). The moduli spaces of the SQCD theories have in this way been realized geometrically in the brane language. Initially interest focused on realizing the electro-magnetic dualities of $\mathrm{N}=1$ SQCD geometrically in these configurations [3]- 6]. The connection was then made between the brane configurations and the IR solutions of N=2 SQCD [4] [7]- 9]; in particular, Witten [7] realized that by extending the decription of the type IIA configuration to Mtheory, and hence providing a description of the strong coupling regime of the type IIA theory, the curve describing the M-theory configuration was precisely that describing the IR physics of the gauge theory. This techique has then been extended to provide derivations of the superpotentials of confining $\mathrm{N}=1 \mathrm{SQCD}$ theories [10] [11]. As proposed in [6] the success of these methods may be thought of as analogous to the strong coupling expansion on the lattice. As one moves to M-theory from the Type IIA theory the eleventh compactified dimension's radius is increased from zero and its associated Kaluza Klien modes fall in mass from infinity [10]. Simultaneously the UV coupling increases and the M-theory configuration provides a semi-classical solution of the strongly coupled model. The solutions are thus for field theories with extra states but which we hope nevertheless lie in the same universality class as the SQCD theories we wish to study. The non-renormalization theorems of supersymmetric theories preserve the semi-classical results into the quantum theory.

The latest success of the string theory approach has been to identify the SQCD string [10] and the confined bound states [12] in the (M-theory modified) SQCD theories with M-theory branes ending on the configurations surface. The M-theory predictions for string tensions are in agreement with the field theory results when $\mathrm{N}=2 \mathrm{SQCD}$ is broken to $\mathrm{N}=1$ [12]. The success in qualitatively identifying the theories bound states in some sense supersedes the field theory successes.

The toughest challenge for these new methods is to move beyond SQCD to non-supersymmetric theories. Some progress has been made within the context of field theory in studying the SQCD solutions in the presence of perturbing soft supersymmetry breaking interactions [17]-25] such as a gaugino mass. These breakings are introduced through the vevs of the auxilliary components of spurion coupling fields [17, a technique that allows the symmetries of the SQCD theories to be preserved into the non-supersymmetric regime. Unfortunately the decoupling limit for the super partners may not be taken, in N=1 SQCD because of unknown Kahler terms that contribute directly to the potential of the softly 
broken theories [18], and in N=2 SQCD because of the restrictions of the derivative expansion of the SQCD solution enforcing an expansion in the breaking terms over $\Lambda$ [19] [21]. Nevertheless these softly broken theories exhibit different behaviour to the SQCD theories. The soft breakings break the $Z_{N}$ symmetry of SQCD and the theories have a unique vacuum. They also display $\theta$-angle dependence [18] [20], typically having phase transitions at $\theta_{\text {phys }}=($ odd $) \pi$.

Non-supersymmetric D-brane configurations have been proposed in Type IIA string theory [5] [10] and Witten has provided a curve describing the M-theory extension of that configuration. The first attempt at a quantitative understanding of this set up has appeared recently in [12]. The authors again construct M-theory branes connecting the surface that may be interpreted as QCD strings and semi-classically calculate the string tensions. They suggest that the ratios of string tensions found in the N=1 SQCD theories might carry over to the $\mathrm{N}=0$ theory.

In this paper we propose an identification of the field theory on this $\mathrm{N}=0$ brane configuration. We expect the breaking of $\mathrm{N}=2$ supersymmetry in 10D string theory by the string dynamics to appear as spontaneous breaking in the low energy field theory description. Non-renormalizable operators will be suppressed by the scale of the breaking (here given by the brane tensions and hence of order the Planck mass). Thus any breakings in the low energy theory will be precisely of the form of soft breakings that may be introduced through the vevs of auxilliary spurion fields. The $\mathrm{N}=2$ theory has a single spurion coupling, the gauge coupling $\tau$ which is a member of an $\mathrm{N}=2$ spurion multiplet and hence there is a unique fashion in which the soft breakings may enter the theory. We show that the induced breakings correctly leave the field content described by the brane configuration.

The identification of the string theory configurations with softly broken SQCD for which field theory solutions exist (for small breakings) allows us to test the quantitative prediction of M-theory for the string tensions in these models. We explicitly calculate the string tensions in the $\mathrm{SU}(\mathrm{N})$ theory as one moves away from the supersymmetric point. The semi-classical M-theory prediction is found to be only the zeroth order contribution to the ratio of string tensions which have additional contributions that can be expanded in powers of the soft breaking parameter. We evaluate the ratios to first order in that breaking parameter.

\section{Review of $\mathrm{N}=2$ and $\mathrm{N}=1$ Configurations}

We begin with a brief overview of the brane configurations describing supersymmetric field theories (in this paper we restrict our attention to models without matter fields). The 
constructions all consist of Type IIA D4-branes suspended between NS5 branes which lie at various angles to each other.

\section{$2.1 \mathrm{~N}=2 \mathrm{SQCD}$}

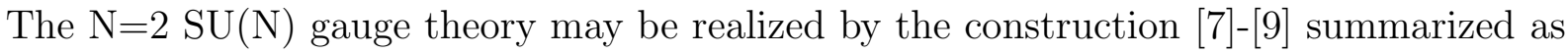

\begin{tabular}{|c|c|c|c|c|c|c|c|c|}
\hline & $\#$ & $R^{4}$ & $x^{4}$ & $x^{5}$ & $x^{6}$ & $x^{7}$ & $x^{8}$ & $x^{9}$ \\
\hline $\mathrm{NS}$ & 2 & - & - & - & $\bullet$ & $\bullet$ & $\bullet$ & $\bullet$ \\
\hline $\mathrm{D} 4$ & $N_{c}$ & - & $\bullet$ & $\bullet$ & {$[-]$} & $\bullet$ & $\bullet$ & $\bullet$ \\
\hline
\end{tabular}

$R^{4}$ is the space $x^{0}-x^{3}$. A dash - represents a direction along a brane's world wolume while a dot $\bullet$ is transverse. For the special case of the D4-branes' $x^{6}$ direction, where the world volume is a finite interval corresponding to its suspension between the two NS5 branes at different values of $x^{6}$, we use the symbol [-].

The configuration has $\mathrm{N}=2$ supersymmetry in four dimensions. The $4 \mathrm{D}$ gauge theory is realized on the $R^{4}$ world volume of the D4 branes. Strings connecting the $N_{c}$ D4-branes give rise to gauge bosons on the D4-branes' world volumes. When the D4-branes are coincident the gauge group is an unbroken $S U\left(N_{c}\right)$ [7]. The D4-branes can be separated in the $x^{4}$ and $x^{5}$ directions in which case the elementary strings are no longer of zero length and so correspond to massive gauge fields. In general when the D4-branes are separated the gauge symmetry is $U(1)^{N_{c}-1}$. Hence this motion is the geometrical representation of the higgs mechanism associated with an adjoint scalar in the $\mathrm{N}=2$ theory. The field theory exists on scales much greater than the $L_{6}$ distance between the NS5 branes with the fourth space like direction of the D4-branes generating the coupling of the gauge theory in the effective 4D theory.

Witten's IR solution of the model [7] comes from extending the configuration to Mtheory by bringing the compactification radius, $R$, of the eleventh dimension $x^{10}$ up from zero. Whilst the type IIA string theory description of the D4-branes ending on the NS5 branes is a strongly coupled problem it has a smooth M-theory description since both the D4-branes and NS5 branes are aspects of a single M-theory 5-brane but which in places is wrapped around the compact $x^{10}$ direction. Using the compact notation

$$
v=x^{4}+i x^{5}, \quad t=\exp \left(x^{6}+i x^{10}\right) / R
$$

The holomorphic curve $\Sigma$ describing the field theory configuration above is

$$
t^{2}+B(v) t+1=0
$$


where $B(v)$ is a polynomial of order $N_{c}$ in $v$. The curve is precisely that of the form of the IR solution of the $\mathrm{N}=2$ field theory.

Recall that the $\mathrm{N}=2$ field theory [14]-16] has a quantum moduli space corresponding to different choices of the adjoint vev. The gauge symmetry is generically broken down from $S U\left(N_{c}\right)$ to $U(1)^{N_{c}-1}$ and the neutral components of the adjoint matter field remain massless. There are $N_{c}$ points on the moduli space with $N_{c}-1$ massless monopoles or dyons. At each point, in the basis where all the local low energy $\theta$ angles are set to zero, there are $N_{c}-1$ massless monopoles each charged only under a single $U(1)$ factor. The electric variables are strongly coupled about these points but the electro-magnetic duality of the theory provides a weakly coupled dual description of the physics about each point. The electric and magnetic variables for the $N_{c}-1$ massless components of the adjoint matter field, $a_{i}$ and $a_{D i}$ are given by period integrals of the curve (3). These periods can be translated to the more useful form of a prepotential appropriate to the local patch of the moduli space [16]. For example the theory close to a singular point is described by the Lagrangian

$$
\begin{aligned}
\mathcal{L}= & \sum_{i}^{N_{c}-1}\left(\frac{1}{4 \pi} \operatorname{Im}\left[\int d^{4} \theta \frac{\partial \mathcal{F}_{\mathrm{eff}}}{\partial A_{D i}} \bar{A}_{D i}+\frac{1}{2} \int d^{2} \theta \frac{\partial^{2} \mathcal{F}_{\mathrm{eff}}}{\partial A_{D i}^{2}} W^{i} W^{i}\right]\right. \\
& \left.+\int d^{4} \theta\left(M_{i}^{\dagger} e^{2 V_{D i}} M_{i}+\tilde{M}_{i}^{\dagger} e^{-2 V_{D i}} \tilde{M}_{i}\right)+2 \sqrt{2} \operatorname{Re} \int A_{D i} M_{i} \tilde{M}_{i}\right)
\end{aligned}
$$

where $\mathcal{F}_{\text {eff }}$ is the prepotential [16]

$$
\mathcal{F}_{\text {eff }}=-i \frac{N_{c}^{2} \Lambda^{2}}{2 \pi}-\frac{2 N_{c} \Lambda}{\pi} \sum_{k=1}^{N_{c}-1} s_{k} A_{D k}-\frac{i}{4 \pi} \sum_{k=1}^{N_{c}-1} a_{D k}^{2} \ln \frac{a_{D k}}{\Lambda_{k}}+\mathcal{O}\left(a_{D}^{3}\right)
$$

with

$$
s_{k}=\sin \frac{k \pi}{N_{c}}, \quad \ln \frac{\Lambda_{k}}{\Lambda}=\frac{3}{2}+\ln s_{k}
$$

and

$$
\Lambda=\Lambda_{U V} e^{i 2 \pi \tau_{0} / b_{0}}, \quad \tau_{0}=\frac{\theta_{0}}{2 \pi}+i \frac{4 \pi}{g_{0}^{2}}
$$

where $b_{0}=2 N_{c}$ is the one loop $\beta$ function coefficient and $g_{0}$ the UV coupling at scale $\Lambda_{U V}$.

We note that at higher order in $a_{D k}$ the $U(1)$ factors begin to mix in the IR effective theory (in all calculations below we will work at lower order and neglect this complication).

\section{$2.2 \mathrm{~N}=1 \mathrm{SQCD}$}

$\mathrm{N}=1$ SQCD is realized very similarly to the $\mathrm{N}=2$ configuration but by simply rotating one of the NS5 branes into the $x^{8}-x^{9}$ directions. There is in fact a continuous set of $\mathrm{N}=1$ configurations [8] associated in the brane language with rotating the " $v$-plane" of one NS5 
brane in the $\mathrm{N}=2$ configuration into the $x^{8}-x^{9}$ directions. In the field theory this is interpreted as smoothly turning on a mass for the adjoint matter field of the $\mathrm{N}=2$ model. The configuration is now

\begin{tabular}{|c|c|c|c|c|c|c|c|c|}
\hline & $\#$ & $R^{4}$ & $x^{4}$ & $x^{5}$ & $x^{6}$ & $x^{7}$ & $x^{8}$ & $x^{9}$ \\
\hline NS & 1 & - & - & - & $\bullet$ & $\bullet$ & $\bullet$ & $\bullet$ \\
\hline NS & 1 & - & $\bullet$ & $\bullet$ & $\bullet$ & $\bullet$ & - & - \\
\hline D4 & $N_{c}$ & - & $\bullet$ & $\bullet$ & {$[-]$} & $\bullet$ & $\bullet$ & $\bullet$ \\
\hline
\end{tabular}

The IR superpotential is again obtained by moving to the M-theory description [10] in which the curve is described by (with $w=x^{8}+i x^{9}$ )

$$
t=v^{N_{c}}, \quad v w=\xi
$$

For large $v, w \simeq 0$ and $\Sigma$ has a $Z_{N_{c}}$ symmetry but in the interior of the curve the symmetry is broken by the second equation in (9). The IR superpotential, $W=N_{c} \Lambda^{3} \simeq 4 \pi i R N_{c} \xi$ may be obtained from $\Sigma$ by performing a volume integral in $v, w$, ln $t$ space over an appropriate volume with $\Sigma$ as one surface (see [10]).

\section{$3 \quad \mathrm{~N}=0$ Branes And Field Theory Identification}

Our interest here is in the possibility that these brane configurations may be able to shed light on non-supersymmetric configurations. We will again only consider the matter free case in the hope of identifying the resulting field theories. As pointed out in [10] an arbitrary rotation of the $v$-plane of one NS5 brane of the $\mathrm{N}=2$ configuration in the $x^{4}, x^{5}, x^{7}, x^{8}, x^{9}$ volume leads to an $\mathrm{N}=0$ configuration. There are 6 degrees of freedom $(S O(5) / S O(2) \times S O(3))$ associated with these rotations. Witten has provided a discription of the M-theory configuration corresponding to models with arbitrary rotations of the $v$-plane in the $x^{4}, x^{5}, x^{7}, x^{8}$ volume (we write these four coordinates as the vector $\vec{A}$ ). It is a minimal area embedding solution and takes the form

$$
\begin{aligned}
\vec{A} & =\operatorname{Re}\left(\vec{p} \lambda+\vec{q} \lambda^{-1}\right) \\
x^{6} & =-c R N_{c} \operatorname{Re} \ln \lambda \\
x^{10} & =-N_{c} \operatorname{Im} \ln \lambda
\end{aligned}
$$

where $\vec{p}$ and $\vec{q}$ are complex four vectors and with $c$ are chosen to satisfy the Virasoro constraint

$$
\vec{p}^{2}=\vec{q}^{2}=0, \quad-\vec{p} \cdot \vec{q}+\frac{R^{2} N_{c}^{2}}{2}\left(1-c^{2}\right)=0
$$


The supersymmetric configurations of (11) and (8) are realized by the choices: $\vec{p}=$ $(1, i, 0,0), \vec{q}=(1, i, 0,0), c= \pm 1(\mathrm{~N}=2)$, and $\vec{p}=(1, i, 0,0), \vec{q}=(0,0,1, i), c= \pm 1(\mathrm{~N}=1)$. In addition the $\mathrm{N}=0$ configuration of [5] may be reproduced $(\vec{p}=(1, i, 0,0), \vec{q}=(1,0, i, 0), c=$ $\left.\pm\left(1-2 / N_{c}^{2} R^{2}\right)^{1 / 2}\right)$

\begin{tabular}{|c|c|c|c|c|c|c|c|c|}
\hline & $\#$ & $R^{4}$ & $x^{4}$ & $x^{5}$ & $x^{6}$ & $x^{7}$ & $x^{8}$ & $x^{9}$ \\
\hline $\mathrm{NS}$ & 1 & - & - & - & $\bullet$ & $\bullet$ & $\bullet$ & $\bullet$ \\
\hline $\mathrm{NS}$ & 1 & - & - & $\bullet$ & $\bullet$ & - & $\bullet$ & $\bullet$ \\
\hline $\mathrm{D} 4$ & $N_{c}$ & - & $\bullet$ & $\bullet$ & {$[-]$} & $\bullet$ & $\bullet$ & $\bullet$ \\
\hline
\end{tabular}

This configuration describes a non-supersymmetric $S U\left(N_{c}\right)$ gauge theory with a real adjoint scalar field corresponding to the freedom to separate the D4-branes in the $x^{4}$ direction.

Can we identify the supersymmetry breaking terms introduced into the $\mathrm{N}=2$ theory on the brane? The string theory at tree level is a supersymmetric theory. Thus when we find a low energy description in which supersymmetry is broken it can only be because the background field configurations (the branes) have induced supersymmetry breaking through the vevs of fields in the fundamental theory. Supersymmetry breaking is generated by vevs of the auxilliary fields of chiral superfields. The low energy supersymmetry breaking parameters are therefore given by these field vevs in a low energy description in which oscillations about those vevs are ignored. Such fields are called spurion couplings in the field theory. A detailed discussion of this mechanism in string theory where the supersymmetry breaking is the result of a choice of compactification is in [21]. In the brane picture these fields also have explicit realizations. For example the gauge coupling is given by the $x^{6}$ distance between the two NS5 branes which can equally be thought of as the vev of a scalar field living on the branes (describing the brane positions). The oscillations of the scalar are neglected since the branes are so heavy that any oscillation in the $x^{6}$ direction is negligible in the low energy field theory.

If we do not neglect those oscillations then the coupling will appear in the field theory as a field. This scalar as part of a superfield will have superpartners. For the $\mathrm{N}=2$ configuration these partners will fill out an $\mathrm{N}=2$ multiplet. In fact as discussed in 21 there is a unique way in which the coupling may be included in the field theory as a spurion field. It must appear as a vector multiplet corresponding to a $U(1)$ symmetry [21]. In fact in complete generality there can be two spurion fields that enter the $4 \mathrm{D}$ field theory prepotential as

$$
\mathcal{F}_{c l}=\frac{N_{c}}{2 \pi}\left(S_{1}+i S_{2}\right) A^{2}
$$

One might be tempted to write $S_{1}$ and $S_{2}$ as sums or products of superfields but the spurion symmetries are such as to constrain those fields to only ever occur in the combinations $S_{1}$ 
and $S_{2}$. One might also include terms of higher dimension in $A$ (the scalar spurion vev which is the coefficient must then be zero in the $\mathrm{N}=2$ limit so these terms vanish) but these terms will generate higher dimension terms in the Lagrangian which are irrelevant for the low energy physics. The Lagrangian is given by

$$
\begin{aligned}
\mathcal{L}= & \frac{1}{4 \pi} \operatorname{Im}\left[\int d^{4} \theta\left(\frac{\partial \mathcal{F}_{c l}}{\partial A} \bar{A}+\frac{\partial \mathcal{F}_{c l}}{\partial S_{1}} \bar{S}_{1}+\frac{\partial \mathcal{F}_{c l}}{\partial S_{2}} \bar{S}_{2}\right)\right. \\
& +\int d^{2} \theta\left(\frac{1}{2} \frac{\partial^{2} \mathcal{F}_{c l}}{\partial A^{2}} W W+\frac{\partial^{2} \mathcal{F}_{c l}}{\partial A \partial S_{1}} W W^{\prime}+\frac{1}{2} \frac{\partial^{2} \mathcal{F}_{c l}}{\partial S_{1}^{2}} W^{\prime} W^{\prime}\right. \\
& \left.\left.+\frac{\partial^{2} \mathcal{F}_{c l}}{\partial A \partial S_{2}} W W^{\prime \prime}+\frac{1}{2} \frac{\partial^{2} \mathcal{F}_{c l}}{\partial S_{2}^{2}} W^{\prime \prime} W^{\prime \prime}+\frac{\partial^{2} \mathcal{F}_{c l}}{\partial S_{1} \partial S_{2}} W^{\prime} W^{\prime \prime}\right)\right] .
\end{aligned}
$$

Freezing the scalar components of the spurion multiplets' matter fields generates the coupling of the pure glue model with $s_{1}+i s_{2}=\pi \tau_{0} / N_{c}$ (this normalization is convenient since $\Lambda \sim$ $\left.\exp \left(i\left(s_{1}+i s_{2}\right)\right)\right)$.

Since this is the unique way in which a spurion may enter the $\mathrm{N}=2 \mathrm{SQCD}$ theory there is a unique way in which soft breakings may be included. That is by also freezing the complex $F$-components of the spurion matter fields, $F_{1}, F_{2}$, or the real $D$-components of the spurion vector fields, $D_{1}, D_{2}$, and generate soft breaking masses

$$
\begin{gathered}
-\frac{N_{c}}{8 \pi^{2}} \operatorname{Im}\left(\left(F_{1}^{*}+i F_{2}^{*}\right) \psi_{A}^{\alpha} \psi_{A}^{\alpha}+\left(F_{1}+i F_{2}\right) \lambda^{\alpha} \lambda^{\alpha}+i \sqrt{2}\left(D_{1}+i D_{2}\right) \psi_{A}^{\alpha} \lambda^{\alpha}\right) \\
-\frac{N_{c}}{4 \pi^{2} \operatorname{Im}\left(s_{1}+i s_{2}\right)}\left(\left(\left|F_{1}\right|^{2}+D_{1}^{2} / 2\right) \operatorname{Im}\left(a^{\alpha}\right)^{2}+\left(\left|F_{2}\right|^{2}+D_{2}^{2} / 2\right) \operatorname{Re}\left(a^{\alpha}\right)^{2}\right. \\
\left.+\left(F_{1} F_{2}^{*}+F_{1}^{*} F_{2}+D_{1} D_{2}\right) \operatorname{Im}\left(a^{\alpha}\right) \operatorname{Re}\left(a^{\alpha}\right)\right)
\end{gathered}
$$

As there is a unique possibility for how these spurions occur in the field theory the string theory brane configurations have no choice but to break supersymmetry in this fashion. A number of checks can be performed comparing the field theory and brane configuration that seem to bare out this statement. Firstly there are six independent degrees of freedom that break supersymmetry in each case. In the field theory $F_{1}, F_{2}, D_{1}, D_{2}$. In the brane configuration there are six independent rotations of one of the NS5 branes that break $\mathrm{N}=2$ supersymmetry to $\mathrm{N}=0$ as can be seen from (1) and (12); the $x^{4}$ dimension into each of $x^{7}, x^{8}, x^{9}$ or the $x^{5}$ dimension into $x^{7}, x^{8}, x^{9}$. These rotations each leave a real scalar degree of freedom massless corresponding to the ability to shift D4 branes in the unrotated one of those two coordinates. Encouragingly switching on any one of the field theory parameters also leaves a massless scalar as can be observed in (15). The identification between the brane picture and the field theory is therefore that each of these six rotations corresponds to switching on one of the soft breaking parameters.

In the brane picture rotating both of the $x^{4}, x^{5}$ directions together into the $x^{6}, x^{7}$ plane can be performed in a $\mathrm{N}=1$ supersymmetry preserving way [8]. If our identification is correct 
then this should correspond to switching on two of the soft breaking parameters with equal magnitudes. We indeed find in the field theory that this leaves an $\mathrm{N}=1$ supersymmetric spectrum; for example setting $\operatorname{Re}\left(F_{2}\right)=\operatorname{Im}\left(F_{1}\right)=M$ and all other components zero leaves the gaugino in the field theory massless. The massive fermion and the two components of the scalar have a mass consistent with adding to the $\mathrm{N}=2$ theory a term

$$
\frac{N_{c}}{4 \pi^{2}} \operatorname{Im} \int d^{2} \theta M A^{2}
$$

These non-trivial consistency checks strongly suggest that the field theory identification is correct.

\section{The QCD Strings}

Witten [10] has identified the SQCD string in the M-theory broadened configurations corresponding to $\mathrm{N}=1 \mathrm{SQCD}$ with M-theory two-branes ending on $\Sigma$ so they appear as strings in $R^{4}$. They will take the form of a one-brane on $R^{4}$ "crossed" with a one-brane outside $R^{4}$ with each end on $\Sigma$. The string outside $R^{4}$ can be described by

$$
\begin{aligned}
v & =\sqrt{\xi} t_{0}^{1 / N_{c}} \exp \left(2 k \pi i \sigma / N_{c}\right) \\
w & =\xi v^{-1}
\end{aligned}
$$

where $t_{0}^{1 / N_{c}}$ is a particular $N_{c}$ th root of $t_{0} . \sigma$ is a coordinate along the string and runs from 0 to 1 . For each choice of $k=1 . . N_{c}-1$ the string wraps $k$ times in $x^{10}$ (a string that wraps $N_{c}$ times has been shown by Witten to vanish corresponding to the annihilation of $N_{c}$ mesons into two baryons). The SQCD string tensions are therefore proportional to the mimimal possible length of the string in (17)

$$
\sqrt{|\Delta v|^{2}+|\Delta w|^{2}}=2 \sqrt{\xi} \sqrt{t_{0}^{2 / N_{c}}+t_{0}^{-2 / N_{c}}} \sin \left(\pi k / N_{c}\right)
$$

The minimum of this length is when $t_{0}=1$. These theories therefore have $N_{c}-1$ different SQCD strings with ratios of string tensions 12

$$
\frac{T_{k}}{T_{l}}=\frac{\sin \frac{\pi k}{N_{c}}}{\sin \frac{\pi l}{N_{c}}}
$$

In 12 it has been shown that this result holds for the complete set of $\mathrm{N}=1$ brane configurations obtained by smoothly rotating the $v$-plane of one NS5 brane into the $\mathrm{N}=2$ configuration. This result is in agreement with the calculated string tensions in models where $\mathrm{N}=2 S U\left(N_{c}\right)$ SQCD is broken to $\mathrm{N}=1$ with adjoint matter mass [15]. 
A similar M-theory calculation can be carried out for strings in the $\mathrm{N}=0$ configurations [12]. The QCD strings are again two-branes in the M-theory. By the symmetry of the brane set up the minimal length strings outside $R^{4}$ connecting $\Sigma$ and wrapping $k$ times around $x^{10}$ will lie at $x^{6}=0$ and hence the end points will lie at the points parameterized in (10) by $\lambda=e^{i \sigma_{1}}, e^{i \sigma_{2}}$, with $\sigma_{1}-\sigma_{2}=2 \pi k / N_{c}$. The ratios of string lengths is again given by (19). The authors of [12] proposed that this semi-classical result may hold in the $\mathrm{N}=0$ quantum theory. We test this quantitative prediction in the next section.

\section{$5 \quad \mathrm{~N}=0$ Field Theory Calculation of String Tensions}

We have identified the field theories corresponding to the low energy description of the $\mathrm{N}=0$ brane configurations as $\mathrm{N}=2 \mathrm{SQCD}$ softly broken by the $\mathrm{N}=2 \tau$ spurion vevs. These models have been studied and solved [21]-[23] for small perturbing soft breakings (corresponding to small angle rotations relative to the starting $\mathrm{N}=2$ configuration in the brane picture). We will make use of these solved models to test the M-theory predictions for the ratios of string tensions described above.

The quantum theory of the softly broken models is described by a prepotential that is holomorphic in $A_{i}$ and $S_{j}$ (below we denote $S_{1}=A_{D 1}, S_{2}=A_{D 2}$ and the $N_{c}-1 \mathrm{U}(1)$ sectors by the index $i$ ). The occurence of the spurions is known from the $\mathrm{N}=2$ solutions since their lowest component vevs are simply $\tau_{0}$. That is the prepotential remains that of (5)). Note that $S_{i}$ are globally defined variables while $A_{i}$ and $A_{D i}$ are only locally defined on the $\mathrm{N}=2$ moduli space. The $\mathrm{N}=0$ theory with $N_{c}=2$ has been completely studied [21] and the global minimum lies close to the singular point on the $\mathrm{N}=2$ moduli space with a massless monopole. For larger $N_{c}$ the theory is strongly believed [22] to behave similarly pinning the potential near the singular point with $N_{c}-1$ massless monopoles. We shall study the theory in the local coordinates appropriate to that patch of parameter space. The theory is described by

$$
\begin{aligned}
\mathcal{L}= & \sum_{i, j}\left(\frac{1}{4 \pi} \operatorname{Im} \int d^{4} \theta \frac{\partial \mathcal{F}_{\text {eff }}}{\partial A_{D i}} \bar{A}_{D i}+\frac{1}{8 \pi} \operatorname{Im} \int d^{2} \theta\left(\frac{\partial^{2} \mathcal{F}_{\text {eff }}}{\partial A_{D i} \partial A_{D j}} W_{i} W_{j}\right)\right. \\
& \left.+\int d^{4} \theta M_{i}^{\dagger} e^{2 V_{D i}} M_{i}+\tilde{M}_{i}^{\dagger} e^{-2 V_{D i}} \tilde{M}_{i}+2 \sqrt{2} R e \int A_{D i} M_{i} \tilde{M}_{i}\right)
\end{aligned}
$$

with $\mathcal{F}_{\text {eff }}$ given by (5). Performing the superspace integrals [22] we obtain the potential

$$
\begin{aligned}
V= & \sum_{i}\left[\frac{1}{2 b_{i i}}\left(\left|m_{i}\right|^{2}+\left|\tilde{m}_{i}\right|^{2}\right)^{2}+2\left|a_{D i}\right|^{2}\left(\left|m_{i}\right|^{2}+\left|\tilde{m}_{i}\right|^{2}\right)\right. \\
& +\frac{1}{b_{i i}}\left(\sqrt{2}\left(b_{1 i} F_{1}^{*}+b_{2 i} F_{2}^{*}\right) m_{i} \tilde{m}_{i}+\sqrt{2}\left(b_{1 i} F_{1}+b_{2 i} F_{2}\right) \bar{m}_{i} \bar{m}_{i}\right) \\
& +\frac{1}{b_{i i}}\left(b_{1 i} D_{1}+b_{2 i} D_{2}\right)\left(\left.m_{i}\right|^{2}-\left|\tilde{m}_{i}\right|^{2}\right)
\end{aligned}
$$




$$
\begin{aligned}
& \left.+\frac{1}{b_{i i}}\left(b_{1 i}^{2}\left(\left|F_{1}\right|^{2}+\frac{1}{2} D_{1}^{2}\right)+b_{2 i}^{2}\left(\left|F_{2}\right|^{2}+\frac{1}{2} D_{2}^{2}\right)+b_{1 i} b_{2 i}\left(F_{1} F_{2}^{*}+F_{1}^{*} F_{2}+D_{1} D_{2}\right)\right)\right] \\
& -b_{11}\left(\left|F_{1}\right|^{2}+\frac{1}{2} D_{1}^{2}\right)-b_{22}\left(\left|F_{2}\right|^{2}+\frac{1}{2} D_{2}^{2}\right)-b_{12}\left(F_{1} F_{2}^{*}+F_{1}^{*} F_{2}+D_{1} D_{2}\right)
\end{aligned}
$$

where

$$
b_{i j}=\frac{1}{4 \pi} \operatorname{Im} \frac{\partial^{2} \mathcal{F}_{\mathrm{eff}}}{\partial a_{i} \partial a_{j}}
$$

For simplicity we set the spurion components $D_{1}=D_{2}=0$. Minimizing with respect to $m_{i}$ and $\tilde{m}_{i}$ leads to the result (see [22] for details)

$$
\begin{aligned}
V= & -b_{11}\left|F_{1}\right|^{2}-b_{22}\left|F_{2}\right|^{2}-b_{12}\left(F_{1}^{*} F_{2}+F_{1} F_{2}^{*}\right) \\
& +\sum_{i}\left[\frac{b_{1 i}^{2}}{b_{i i}}\left|F_{1}\right|^{2}+\frac{b_{2 i}^{2}}{b_{i i}}\left|F_{2}\right|^{2}+\frac{b_{1 i} b_{2 i}}{b_{i i}}\left(F_{1}^{*} F_{2}+F_{1} F_{2}^{*}\right)-2 \frac{\rho_{i}^{4}}{b_{i i}}\right]
\end{aligned}
$$

and

$$
\rho_{i}^{2}=\left|m_{i}\right|^{2}=\left|\tilde{m}_{i}\right|^{2}=-b_{i i}\left|a_{D i}\right|^{2}-\frac{\left|b_{1 i} F_{1}+b_{2 i} F_{2}\right| \epsilon_{ \pm}}{\sqrt{2}}
$$

where $\epsilon_{ \pm}$is dynamically determined between its two possible values \pm 1 .

We may now find the minimum of the potential using the form of the prepotential in (5). For most choices of soft breakings and $\Lambda$ it is sufficient to work to order $\left|a_{D i}\right|^{2}$ to find the minimum. For special choices of parameters, for example taking $\Lambda$ real $(\theta=0)$ and only $F_{2} \neq 0$, the $\left|a_{D i}\right|^{2}$ terms in the potential can be made to vanish and one must go to order $\left|a_{D i}\right|^{4}$. It is sufficient for displaying the behaviour of the models to work at order $\left|a_{D i}\right|^{2}$ and we find

$$
\left\langle a_{D i}\right\rangle=\frac{i \Lambda}{4 \sqrt{2}} \frac{\left(\left|F_{1}\right|^{2}-\left|F_{2}\right|^{2}+i\left(F_{1}^{*} F_{2}+F_{1} F_{2}^{*}\right)\right)}{\left(\left|\operatorname{Re}(\Lambda) F_{1}-\operatorname{Im}(\Lambda) F_{2}\right|\right)}
$$

Let us now consider some special cases of this result. Firstly when $F_{2}=i F_{1}=i M$ the tree level theory was $\mathrm{N}=1$ supersymmetric. The IR solution is just the familiar result of [15], $\left\langle a_{D i}\right\rangle=0$ (which can be shown to all orders from (23)) and hence

$$
\rho_{i}=\frac{\sqrt{2} N_{c}}{4 \pi^{2}} s_{i} \Lambda^{*} M
$$

The string tensions are given by [26]

$$
T_{k}=2 \pi\left|\left\langle m_{k} \tilde{m}_{k}\right\rangle\right|=2 \pi\left|\rho_{k}\right|
$$

and are proportional to $\sin \left(k \pi / N_{c}\right)$ in agreement with the M-theory calculation (19).

We may also calculate the string tensions in the non-supersymmetric theories. As an example we take $\left|F_{1}\right|=f_{1}, F_{2}=0$ and $\theta=0$. These theories correspond to those in the string theory where the $v$-plane of one NS5 brane is rotated between the configurations of 
(11) and (12). We can of course only keep control of the solution for small soft breakings corresponding to small angles of rotation away from the $\mathrm{N}=2$ configuration. The minimum of the potential corresponds to

$$
\left\langle a_{D i}\right\rangle=\frac{i f_{1}}{4 \sqrt{2}}, \quad \rho_{i}^{2}=\frac{\sqrt{2} N_{c} \Lambda f_{1} s_{i}}{4 \pi^{2}}+\frac{f_{1}^{2}}{64 \pi^{2}}+\frac{f_{1}^{2}}{256 \pi^{2}} \ln \left(f_{1} / 4 \sqrt{2} s_{i} \Lambda\right)
$$

The resulting ratios of string tensions is

$$
\frac{T_{k}}{T_{l}}=\frac{s_{k}}{s_{l}}+\frac{\sqrt{2}}{32 N \Lambda} \frac{s_{l}-s_{k}}{s_{l}^{2}}\left[1+\frac{1}{4} \ln \frac{f_{1}}{4 \sqrt{2} \Lambda}+\frac{1}{4} \frac{s_{k} \ln s_{l}-s_{l} \ln s_{k}}{s_{l}-s_{k}}\right] f_{1}+\mathcal{O}\left(f_{1}^{2}\right) .
$$

The string tension prediction approaches the semi-classical M-theory prediction as $f_{1} \rightarrow 0$ but is renormalized in the softly broken theory. This is perhaps not so great a surprise. As the authors of [12 point out, the semi-classical results are correct in the supersymmetric theories because supersymmetry endows these quantities with a non-renormalization property. There is no such property in the softly broken theories and a priori one should not expect the semiclassical calculation to hold in the non-supersymmetric theory. In some sense though we view (29) as a success of the semi-classical M-theory calculation since the zeroth order term is the M-theory result.

\section{Conclusions}

We have argued that $\mathrm{N}=0 \mathrm{D}$-brane configurations recently proposed in the literature correspond to softly broken $\mathrm{N}=2$ SQCD where the soft breakings result from expectation values of the $\mathrm{N}=2$ spurion coupling $\tau$. These theories are solvable for small soft breakings (corresponding to small rotations away from the $\mathrm{N}=2$ configuration in the brane language). A semi-classical calculation of the string tensions in the theory can be made from the M-theory and agrees with the supersymmetric field theory solutions. The equivalent calculation for $\mathrm{N}=0$ theories has been performed in [12] and proposed as the correct description of the quantum theory. We stress that any such predictions are testable in quantitatively solved non-supersymmetric field theories. We have explicitly calculated the ratio of string tensions in the $\mathrm{N}=0$ field theories showing that at leading order in the soft breaking the $\mathrm{N}=0$ models

renormalize the M-theory result. Unfortunately this result seems to put pay to any hope of obtaining quantitative results about QCD from the M-theory construction.

Finally we comment on possible lattice tests of (29) [25] [27]. Unfortunatly these will be difficult. Lattice regularization explicitly breaks supersymmetry and, while one might hope to recover supersymmetry in the continuum limit, in practice one recovers the model with all possible breaking terms allowed by the gauge symmetry. For pure glue N=1 SQCD 
the only possible such term is a gaugino mass which is a soft breaking of supersymmetry [28. In the $\mathrm{N}=2$ theory though there is no such promise and we expect explicit hard breakings to be present. To obtain the softly broken theories discussed above would require the tuning of many bare lattice operators. Tuning to the supersymmetric point would be equally troublesome but were it identified one might hope to see the string tensions tending to the M-theory ratio (our zeroth order result) as that point is approached.

Acknowledgements: The authors would like to thank R. Sundrum and S. Hsu for insightful comments on the manuscript. This work was in part funded by the DOE under contract numbers DE-AC02-ERU3075 and DE-FG02-92ER40704 and by the NSF under grant number NSF-PHY-94-23002. 


\section{References}

[1] A. Hanany and E. Witten, hep-th/9611230, Nucl. Phys. B492, 152 (1997).

[2] J. de Boer, K. Hori, Y. Oz and Z. Yin, hep-th/9702154, Nucl. Phys. B502, 107 (1997); O. Aharony, A. Hanany, hep-th/9704170, Nucl. Phys. B504, 239 (1997).

[3] S. Elitzur, A. Giveon and D. Kutasov, hep-th/9702014, Phys. Lett. 400B, 269 (1997);

S. Elitzur, A. Giveon, D. Kutasov, E. Rabinovici and A. Schwimmer, hep-th/9704104, Nucl. Phys. B505, 202 (1997); J. H. Brodie and A. Hanany, hep-th/9704043, Nucl. Phys. B506, 157 (1997); R. Tatar, hep-th/9704198; C. Ahn, K. Oh, R. Tatar, hepth/9707027; C. Csaki and W. Skiba, hep-th/9708082, Phys. Lett. 415B, 31 (1997).

[4] N. Evans, C.V. Johnson and A.D. Shapere, hep-th/9703210, Nucl. Phys. B505, 251 (1997).

[5] A. Brandhuber, J. Sonnenschein, S. Theisen, S. Yankielowicz, hep-th/9704044, Nucl. Phys. B502, 125 (1997).

[6] M. Schmaltz and R. Sundrum, hep-th/9708015.

[7] E. Witten, hep-th/9703166, Nucl. Phys. B500, 3 (1997).

[8] J. L. F. Barbón, hep-th/9703051, Phys. Lett. 402B, 59 (1997).

[9] K. Landsteiner, E. Lopez, and D.A. Lowe, hep-th/9705199, Nucl. Phys. B507, 197 (1997); A. Brandhuber, J. Sonnenschein, S. Theisen and S. Yankielowicz, hepth/9705232, Nucl. Phys. B504, 175 (1997); T. Nakatsu, K. Ohta, T. Yokono and Y. Yoshida, hep-th/9707258.

[10] E. Witten, Nucl. Phys. B507, 658 (1997), hep-th/9706109.

[11] K. Hori, H. Ooguri and Y. Oz, hep-th/9706082; A. Brandhuber, N. Itzhaki, V. Kaplunovsky, J. Sonnenschein and S. Yankielowicz, hep-th/9706127, Phys. Lett. 410B, 27 (1997); S. Nam, K. Oh and S.-J. Sin, hep-th/9707247; J. de Boer and Y. Oz hepth/9708044.

[12] A. Hanany, M.J. Strassler and A. Zaffaroni, hep-th/9707244.

[13] S. Katz, C. Vafa, hep-th/9611090, Nucl. Phys. B497, 196 (1997); M. Bershadsky, A. Johansen, T. Pantev, V. Sadov, C. Vafa, hep-th/9612052, Nucl. Phys. B505, 153 (1997); H. Ooguri, C. Vafa, hep-th/9702180, Nucl. Phys. B500, 62 (1997); C. Vafa, B. Zwiebach, hep-th/9701015, Nucl. Phys. B506, 143 (1997); C. Ahn, K. Oh, hep-th/9704061, Phys. 
Lett. 412B, 274 (1997); C.Ahn, hep-th/9705004; C. Ahn, R. Tatar, hep-th/9705106, Phys. Lett. 413B, 293 (1997).

[14] N. Seiberg and E. Witten, hep-th/9407087, Nucl. Phys. B426, 19 (1994); N. Seiberg and E. Witten, hep-th/9408099, Nucl. Phys. B431, 484 (1994); P.C. Argyres and A. Farragi, hep-th/9411057, Phys. Rev. Lett. 73, 3931 (1995); A. Klemm, W. Lerche, and S. Theisen, hep-th/9505150, Int. J. Mod. Phys. A11 1974 (1996).

[15] M.R. Douglas, S.H. Shenker, hep-th/9503163, Nucl. Phys. B447, 296 (1995).

[16] E. D'Hoker and D.H. Phong, hep-th/9701055, Phys. Lett. 397B, 103 (1997).

[17] N. Evans, S.D.H. Hsu and M. Schwetz, hep-th/9503186, Phys. Lett. 355B, 475 (1995).

[18] N. Evans, S.D.H. Hsu and M. Schwetz, hep-th/9703197, Phys. Lett. 404B, 77 (1997).

[19] N. Evans, S.D.H. Hsu, M. Schwetz and S. Selipsky, hep-th/9508002, Nucl. Phys. B456, 205 (1995).

[20] N. Evans, S.D.H. Hsu and M. Schwetz, hep-th/9608135, Nucl. Phys. B484, 124 (1997).

[21] L. Álvarez-Gaumé, J. Distler, C. Kounnas and M. Mariño, hep-th/9604004, Int. J. Mod. Phys. A11 (1996) 4745

[22] L. Álvarez-Gaumé and M. Mariño, hep-th/9606191, Int. J. Mod. Phys. A12 (1997) 975

[23] L. Álvarez-Gaumé, M. Mariño and F. Zamora, hep-th/9703072, hep-th/9707017.

[24] A. Masiero and G. Veneziano, Nucl. Phys. B249, 593 (1985); O. Aharony, J. Sonnenschein, M. E. Peskin, S. Yankielowicz, hep-th/9507013, Phys. Rev. D52, 6157 (1995); E. D'Hoker, Y. Mimura, N. Sakai, hep-ph/9611458, Phys. Rev. D54, 7724 (1996); K. Konishi, hep-th/9609021, Phys. Lett. 392B, 101 (1997).

[25] N. Evans, S.D.H. Hsu and M. Schwetz, hep-th/9707260.

[26] H.B. Nielsen and P. Olesen, Nucl. Phys. B61, 45 (1973)

[27] G. Koutsoumbas and I. Montvay, hep-lat/9612003, Phys. Lett. 398B, 130 (1997); I. Montvay, hep-lat/9607035 Nucl. Phys. B53, Proc. Suppl. (1997) 853.

[28] G. Curci and G. Veneziano, Nucl. Phys. B292, 555 (1987). 